\title{
EXPERIMENTAL AND COMPUTATIONAL STUDIES ON THE PERFORMANCE OF SOLAR TRACKERS UNDER VORTEX SHEDDING, TORSIONAL DIVERGENCE, AND FLUTTER
}

\author{
J. QUINTELA ${ }^{1}$, J. A. JURADO ${ }^{1}$, C. RAPELA ${ }^{1}$, A. J. ÁLVAREZ ${ }^{1}$, M. ROCA ${ }^{1}$, S. HERNÁNDEZ ${ }^{1}$, \\ M. CID MONTOYA ${ }^{1,2}$, J. M. LÓPEZ ${ }^{3}$, A. J. RUIZ ${ }^{3}$, I. MORENO ${ }^{3} \&$ S. JIMÉNEZ ${ }^{3}$ \\ ${ }^{1}$ School of Civil Engineering, Universidade da Coruña, Spain. \\ ${ }^{2}$ Department of Civil and Environmental Engineering and Earth Sciences, University of Notre Dame, USA. \\ ${ }^{3}$ STI Norland, Spain.
}

\begin{abstract}
The current development of renewable energies has originated a number of new structural typologies that are the physical support of the energy production systems. Photovoltaic energy is a very mature source and it is obtained using rows of panels implemented in a longitudinal grillage. Many studies have been carried out in the past with an aim to improve the capacity to obtain electrical power, but another important issue is the need to guarantee the performance of these industrial facilities under the phenomena induced by the turbulent wind flow, taking into account the fact that they are usually built in wide open spaces. This paper describes an extensive research carried out on two configurations of solar trackers by experimental and computational methods. The former was composed of a number of tests of reduced models of segments of the solar trackers, which were carried out in an aerodynamic wind tunnel. The latter consisted of a series of structural analyses worked out through a finite element model of the full panel subjected to aerodynamic and aeroelastic loads. Several angles of attack of the wind flow and a wide range of wind speeds were included in the study. This approach allowed to clearly evaluate the structural and dynamic performance of both the configurations of solar trackers under the most important wind-induced phenomena such as vortex shedding, torsional divergence, and flutter. The paper relates the phases of the study and informs about the more relevant numerical results obtained in the experiments and the computer analysis.
\end{abstract}

Keywords: aerodynamic and aeroelastic loads, finite elements models, flutter, photovoltaic energy, solar trackers, structural analysis, torsional divergence, vortex shedding, wind tunnel test.

\section{INTRODUCTION}

The phenomena that appear in a body immersed in a fluid and the interaction between elastic bodies and fluid flow are a relevant scientific part of fluid dynamics, and several books are devoted to describe such topics in full $[1,2]$. They relate to how vortex shedding, torsional divergence, and flutter, among other wind-induced mechanisms, are generated and the forces and displacements created in the bodies.

Aerospace engineering is the first field where aerodynamic and aeroelasticity disciplines are of great interest, given the need for guaranteeing the appropriate behavior of the vehicle under any circumstance and the convenience of elaborating designs that avoid aircraft instabilities or undesired responses. Thus, several studies on wind-induced phenomena such as flutter [3, 4], torsional divergence [5, 6], and vortex shedding [7, 8] can be found in the literature.

Bridge engineering was also interested in aerodynamics due to the well-known collapse of the suspension Tacoma Narrows Bridge in 1940. This tragic event started a process of revision of the procedures for long span bridge design and the strategies to be implemented for future structures [9]. Currently, long span cable-supported bridges, as suspension or cable stayed, are designed, taking into account a large number of aeroelastic considerations. 
Several researchers have published their findings in flutter studies [10-12], vortex-induced vibrations [13-15], and torsional divergence [16-18].

Both types of structures, aircrafts and long span bridges, have in common two important characteristics: the expensive budget required for their design and construction and the uncertainty in some aspects of the design, for instance, the values of the loads they have to undergo. Thus, it is not strange that more recently, studies considering random values of the loads and use of numerical optimization methodology have been carried out in these typologies [19-24].

The growth of renewable energy systems has generated new classes of constructions and, among them, farms of photovoltaic panels. There are several studies conducted with an aim to increase the efficiency of energy production of these facilities [25-27]; but in addition to that, a crucial issue of these constructions is their correct performance from the structural point of view. They are installed in open spaces in rural locations clearly exposed to wind flow, and therefore are prone to aerodynamic phenomena.

In the most recent version, photovoltaic solar panels are designed as very slender structures with quite a long length and relevant flexibility [28], as they must be able to change the angle with regard to the horizontal plane. Companies of this industrial sector need to be sure of the performance of their products and, consequently, in addition to the usual structural analysis with static of dynamic loads, more sophisticated studies are convenient, and certainly, comprehensive studies with the objective of understanding the behavior of solar panels under aeroelastic phenomena are very beneficial. But this approach has been used quite recently only [29].

This paper presents a number of experimental and computational studies carried out in the University of Coruña with two configurations of solar panels with the same length but different width, and describes the outcome of the research. The experimental part consists of a series of tests carried out in the aerodynamic wind tunnel TUVA using reduced models of the solar panel with the objective of identifying a number of characteristics of the model, namely, the aerodynamic coefficients, the flutter derivatives, and the Strouhal number.

The experiments also give important knowledge about the safety of the solar panel under the lock-in phenomenon. The computational calculations were made using a finite element model of the complete solar panel, and used the information obtained in the wind tunnel to carry out structural analysis to identify the performance of the structure under torsional divergence and flutter.

The studies were conducted for an ample range of angle of attack of the flow and interval of wind speed. Thus, it can be considered that the aerodynamic properties of these constructions have been investigated in full.

The following sections describe the geometry of the solar panels, the aerodynamic wind tunnel where the experiments took place, the characteristics and size of the reduced models, the setup of the tests, and the results obtained that include the findings of the behavior under vortex shedding. Then, the finite element models are presented and the formulation of the analysis to find out the performance of the solar panels under torsional divergence and multimodal flutter are related. The paper ends with some conclusions about the advantages of the classes of test and computer simulations carried out and the adequacy of the solar panels to undergo wind-induced phenomena.

\section{SOLAR TRACKERS' DESCRIPTION}

The two configurations of solar trackers studied in this research are fabricated by the Spanish company, STI Norland, and consist of a typical configuration of rows of 60 panels. From the 

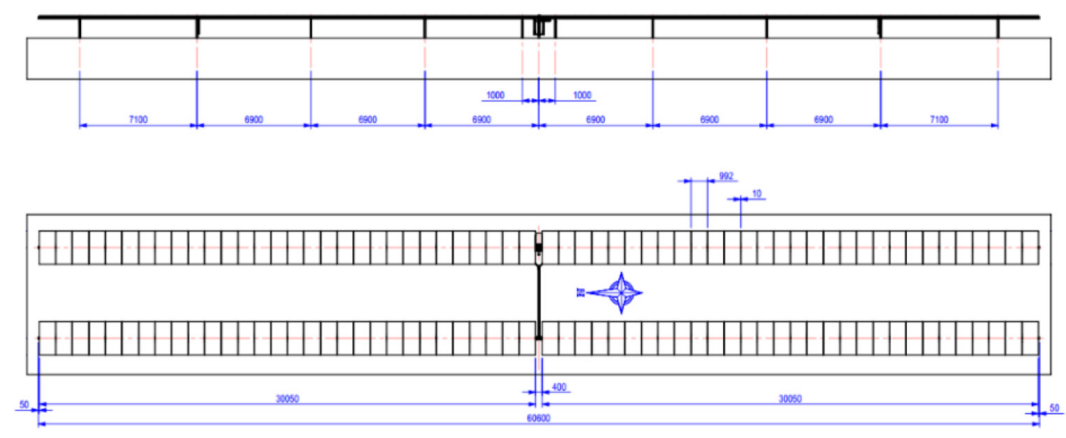

(a) Elevation.

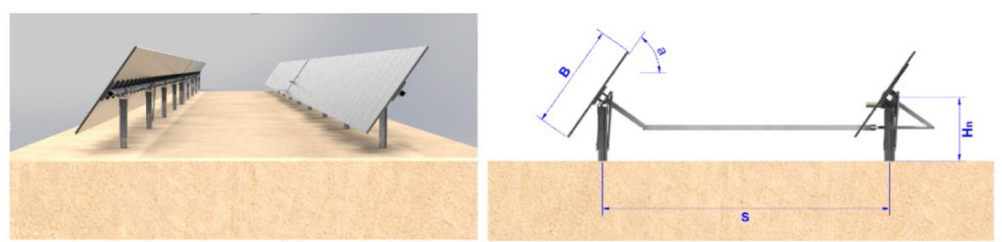

(b) Render of the solar panels and lateral view.

Figure 1: Geometrical definition of the solar trackers.

Table 1: Geometric details of the solar tracker.

\begin{tabular}{llllll}
\hline \multirow{2}{*}{ Geometry } & Width & Height & Pitch & \multicolumn{2}{c}{ Working angle } \\
& $B(\mathbf{m})$ & $H_{n}(\mathbf{m})$ & $s(\mathbf{m})$ & $a_{\min }\left({ }^{\circ}\right)$ & $a_{\text {max }}\left({ }^{\circ}\right)$ \\
\hline Configuration 1 & 2 & 1.2 & 5.5 & -60 & 60 \\
Configuration 2 & 4 & 2 & 7 & -60 & 60 \\
\hline
\end{tabular}

structural point of view, each panel is a rectangular frame that supports the solar cells and is connected to a longitudinal axis that allows the rotation about its direction. The axis is connected to the foundation by vertical struts at intervals of about $7.0 \mathrm{~m}$. In the longitudinal direction, the panels have a length of $1.0 \mathrm{~m}$ and in the transverse direction, the length is 2.0 $\mathrm{m}$ in a configuration and $4.0 \mathrm{~m}$ in the other one. The complete scheme is composed of two rows of panels that are linked by a mechanism that defines the inclination with regards to the horizontal plane, controlled by an actuator located in the central point of the longitudinal axis. Figure 1 shows the geometry of the panels and a computer render of the system.

\section{STRUCTURAL MODEL OF THE SOLAR TRACKERS}

Software SAP2000 was used to define the structural models. The girder and the piers were modeled using bar elements, while the flat panels were modeled as shell elements as shown in Fig. 2. Both rows of the solar tracker have mechanical dampers situated in the second outermost piers acting in the torsional degree of freedom. Each model has 2846 nodes, 3325 bar elements, and 360 shell elements.

Several angles of attack of the solar tracker were considered; therefore, a different structural model for each case was defined. Figures 3 and 4 show the models used for the different cases for each configuration. 

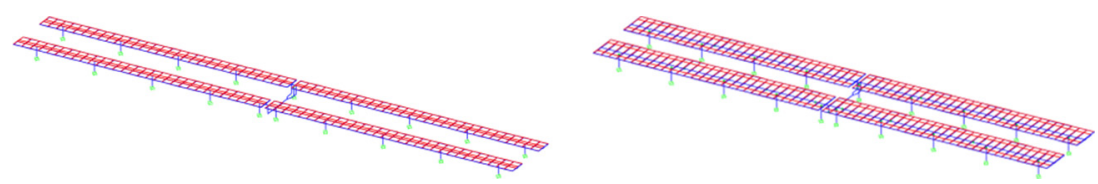

Figure 2: Structural model of the solar trackers for configuration 1 (left) and configuration 2 (right).
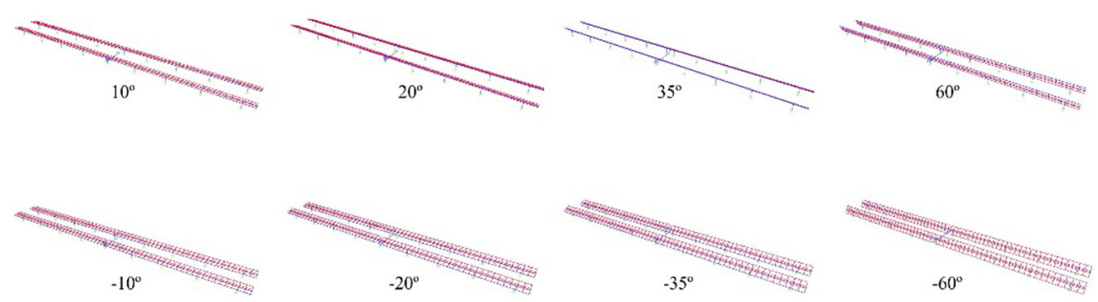

Figure 3: Structural models for negative angles of attack $\left(-10^{\circ},-20^{\circ},-35^{\circ},-60^{\circ}\right)$ and for positive angles of attack $\left(+10^{\circ},+20^{\circ},+35^{\circ},+60^{\circ}\right)$ for configuration 1 .
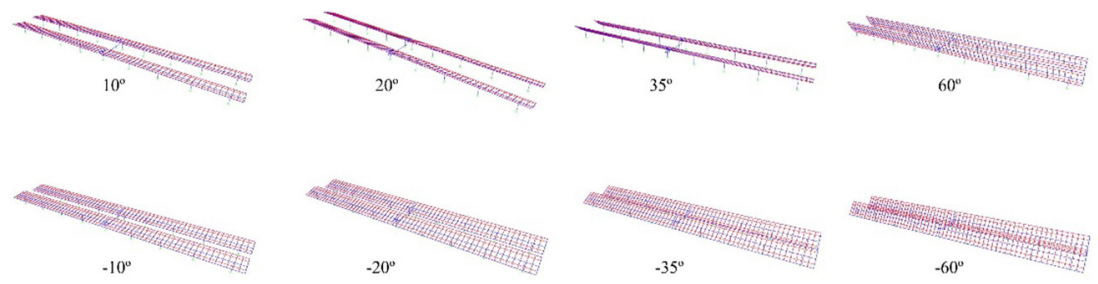

Figure 4: Structural models for negative angles of attack $\left(-10^{\circ},-20^{\circ},-35^{\circ},-60^{\circ}\right)$ and for positive angles of attack $\left(+10^{\circ},+20^{\circ},+35^{\circ},+60^{\circ}\right)$ for configuration 2 .
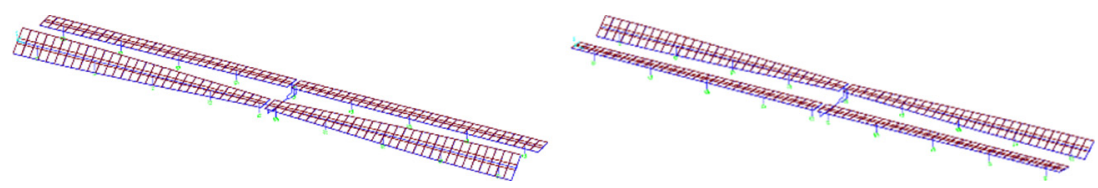

Figure 5: First natural mode shape for each row of the solar tracker.

The natural frequencies and the natural modes were obtained by a modal analysis and later verified with a testing campaign for such purpose over a real-scale tracker prototype. The first four natural modes obtained for every case were torsional, corresponding to the symmetrical and non-symmetrical modes for each row. Figure 5 shows the first mode for each row of the solar trackers.

The first 10 natural frequencies normalized to the first frequency obtained in the modal analysis are shown in Tables 2 and 3. There are no big differences between the natural frequencies obtained for the different angles of attack.

\section{TUVA WIND TUNNEL}

The wind tunnel has an open blowing configuration. It has one fan of Chicago Glower license with a maximum of $1450 \mathrm{rpm}$. It has a rectification piece to transform the circular section to a square section, a diffusor, two meshes, and a contraction element of 6 to 1 with a third-order 
Table 2: Normalized natural frequencies of the first natural modes for configuration 1.

\begin{tabular}{llllllllll}
\hline & \multicolumn{1}{c}{ Angles of attack } \\
Frequencies & $\mathbf{0}^{\circ}$ & $\mathbf{1 0}^{\circ}$ & $\mathbf{- 1 0}^{\circ}$ & $\mathbf{2 0}^{\circ}$ & $\mathbf{- 2 0}^{\circ}$ & $\mathbf{3 5}^{\circ}$ & $\mathbf{- 3 5}^{\circ}$ & $\mathbf{6 0}^{\circ}$ & $\mathbf{- 6 0}^{\circ}$ \\
\hline$\omega_{1}$ & 1.00 & 1.00 & 1.00 & 1.00 & 1.00 & 1.00 & 1.00 & 1.00 & 1.00 \\
$\omega_{2}$ & 1.03 & 1.03 & 1.03 & 1.03 & 1.03 & 1.03 & 1.03 & 1.04 & 1.06 \\
$\omega_{3}$ & 1.04 & 1.03 & 1.03 & 1.03 & 1.03 & 1.03 & 1.03 & 1.04 & 1.06 \\
$\omega_{4}$ & 1.04 & 1.03 & 1.03 & 1.03 & 1.03 & 1.03 & 1.03 & 1.04 & 1.06 \\
$\omega_{5}$ & 2.03 & 2.02 & 2.02 & 2.02 & 2.02 & 2.02 & 2.03 & 2.05 & 2.07 \\
$\omega_{6}$ & 2.04 & 2.03 & 2.03 & 2.02 & 2.03 & 2.03 & 2.03 & 2.05 & 2.07 \\
$\omega_{7}$ & 2.04 & 2.06 & 2.07 & 2.14 & 2.14 & 2.23 & 2.23 & 2.24 & 2.26 \\
$\omega_{8}$ & 2.01 & 2.07 & 2.07 & 2.14 & 2.15 & 2.23 & 2.24 & 2.24 & 2.26 \\
$\omega_{9}$ & 2.53 & 2.52 & 2.52 & 2.52 & 2.52 & 2.53 & 2.53 & 2.56 & 2.59 \\
$\omega_{10}$ & 2.54 & 2.53 & 2.53 & 2.53 & 2.53 & 2.53 & 2.54 & 2.57 & 2.60 \\
\hline
\end{tabular}

Table 3: Normalized natural frequencies of the first natural modes for configuration 2.

\begin{tabular}{llllllllll}
\hline & \multicolumn{1}{c}{ Angles of attack } \\
Frequencies & $\mathbf{0}^{\circ}$ & $\mathbf{1 0}^{\circ}$ & $\mathbf{- 1 0}^{\circ}$ & $\mathbf{2 0}^{\circ}$ & $\mathbf{- 2 0}^{\circ}$ & $\mathbf{3 5}^{\circ}$ & $\mathbf{- 3 5}^{\circ}$ & $\mathbf{6 0}^{\circ}$ & $\mathbf{- 6 0}^{\circ}$ \\
\hline$\omega_{1}$ & 1.00 & 1.00 & 1.00 & 1.00 & 1.00 & 1.00 & 1.00 & 1.00 & 1.00 \\
$\omega_{2}$ & 1.20 & 1.16 & 1.16 & 1.16 & 1.17 & 1.18 & 1.20 & 1.26 & 1.34 \\
$\omega_{3}$ & 1.20 & 1.16 & 1.16 & 1.16 & 1.17 & 1.18 & 1.20 & 1.26 & 1.34 \\
$\omega_{4}$ & 1.20 & 1.16 & 1.16 & 1.16 & 1.17 & 1.18 & 1.20 & 1.26 & 1.34 \\
$\omega_{5}$ & 2.81 & 2.79 & 2.79 & 2.80 & 2.80 & 2.81 & 2.82 & 2.88 & 2.96 \\
$\omega_{6}$ & 3.36 & 3.24 & 3.24 & 3.26 & 3.28 & 3.31 & 3.36 & 3.53 & 3.76 \\
$\omega_{7}$ & 3.36 & 3.24 & 3.24 & 3.26 & 3.28 & 3.31 & 3.36 & 3.53 & 3.76 \\
$\omega_{8}$ & 3.36 & 3.24 & 3.24 & 3.26 & 3.28 & 3.31 & 3.36 & 3.53 & 3.76 \\
$\omega_{9}$ & 3.66 & 3.53 & 3.53 & 3.55 & 3.58 & 3.60 & 3.66 & 3.84 & 4.09 \\
$\omega_{10}$ & 3.80 & 3.67 & 3.67 & 3.68 & 3.71 & 3.74 & 3.80 & 3.99 & 4.25 \\
\hline
\end{tabular}

polynomial shape. This permits a $0.5 \%$ of turbulence level in the test chamber. The test chamber has $1 \mathrm{~m}^{2}$ of section, is $3 \mathrm{~m}$ long, and has a flexible support system of two slider frames in each side of the chamber.

Each frame has a mechanism situated on each side of the chamber which facilitates the change of the angle of attack of the sectional model between $-20^{\circ}$ and $20^{\circ}$. The load cells are situated on these mechanisms, so it is easy to measure the aerodynamic forces as a function of the attack angle. Each lateral wall has a hole of length $2 \mathrm{~m}$ and height $0.2 \mathrm{~m}$, which allows the model axis bar to extend beyond the chamber for supporting. The vertical dimension of the hole makes possible the displacement of the model during the aeroelastic tests to have several centimeters of magnitude. The hole can be covered with magnetic plates to leave free only the required space. Figure 6 shows a general view of the test chamber. 


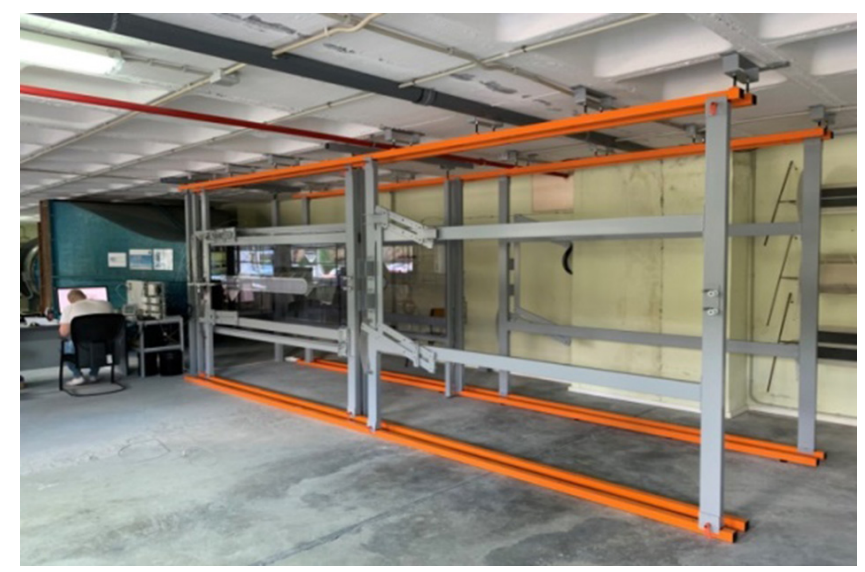

Figure 6: Test chamber of TUVA wind tunnel.

\section{EXPERIMENTAL STUDIES OF THE SOLAR TRACKER IN THE TUVA WIND TUNNEL}

The setup for the experimental studies in the TUVA wind tunnel requires rigid bars for the connection of the sectional models to the load cells. Aerodynamic tests were carried out with the sectional models fixed, while aeroelastic tests require the connection to the load cells with springs allowing displacements in three degrees of freedom.

Wind tunnel control and instrumentation were made by software PCTUVI (Fig. 7). This software allows the calibration of the different parameters of the wind tunnel and the following:

1. carrying out aerodynamic tests of sectional models, obtaining the time history of the forces and moment, and the value of the aerodynamic coefficients;

2. carrying out aeroelastic tests with three degrees of freedom, giving the 18 flutter derivatives by numerical methods [30, 31]; and

3. carrying out vortex shedding tests, giving the time history of the forces or accelerations and the range of the lock-in phenomenon.
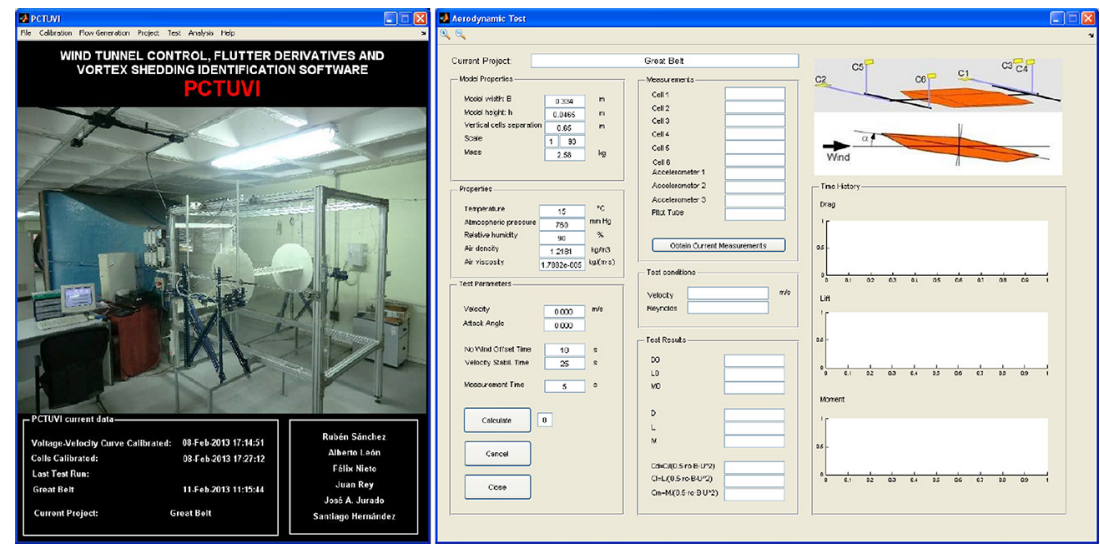

Figure 7: Software PCTUVI. 


\subsection{Identification of the aerodynamic coefficients}

Aerodynamic coefficients can be obtained as a function of the angle of attack $\alpha$ from the aerodynamic forces of drag $(D)$, lift $(L)$, and moment $(M)$ expressed per unit of length as:

$$
C_{D}(\alpha)=\frac{D}{\frac{1}{2} \rho U^{2} B}, \quad C_{L}(\alpha)=\frac{L}{\frac{1}{2} \rho U^{2} B}, \quad C_{M}(\alpha)=\frac{M}{\frac{1}{2} \rho U^{2} B^{2}}
$$

where $\rho$ is the density, $U$ is the mean wind velocity, and $B$ the sectional model width. After testing the models for different wind speeds, the aerodynamic tests were carried out with a wind speed of $9 \mathrm{~m} / \mathrm{s}$. Three different setups were considered to identify the aerodynamic coefficients. Firstly, only one panel of the solar tracker was tested; secondly, one panel was tested modeling the distance to the floor; and finally, two rows of panels with the floor modeled were tested. Results for the three cases are shown in Figs. 8 and 9.

\subsection{Identification of flutter derivatives}

The flutter derivatives were obtained by free vibration aeroelastic tests [32, 33]. The setup for these tests was made by connecting the sectional models to the load cells with 12 springs as shown in Fig. 10.

Springs are characterized by their stiffness, length, and maximum load. The maximum load must be enough to guarantee the linearity of the forces measured. The length has to
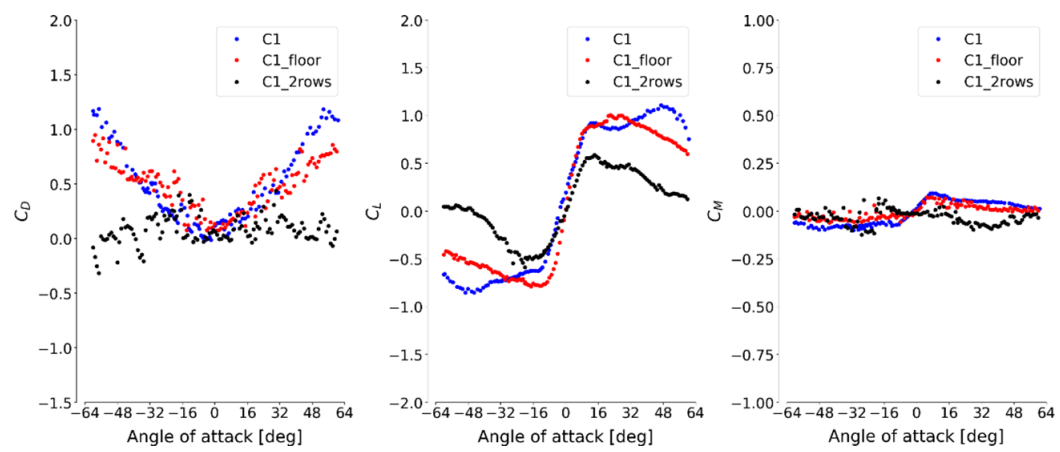

Figure 8: Aerodynamic coefficients for configuration 1.
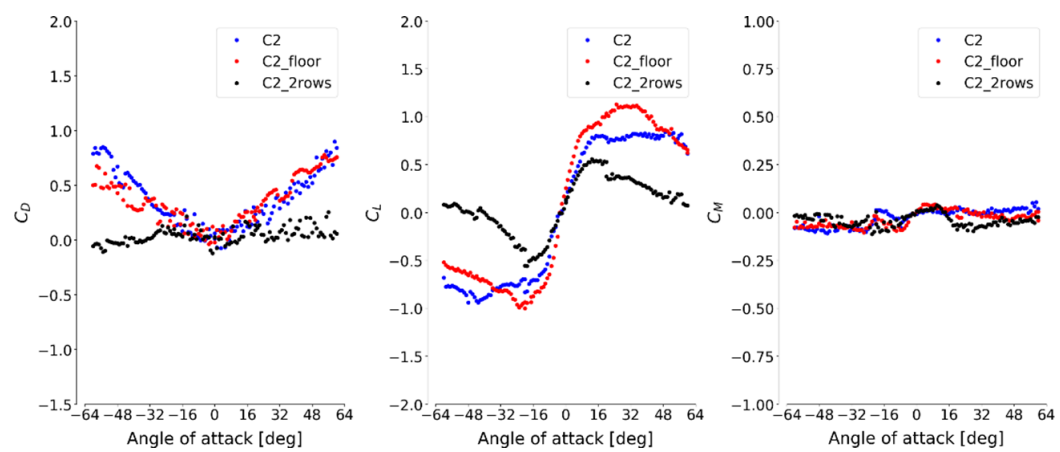

Figure 9: Aerodynamic coefficients for configuration 2. 


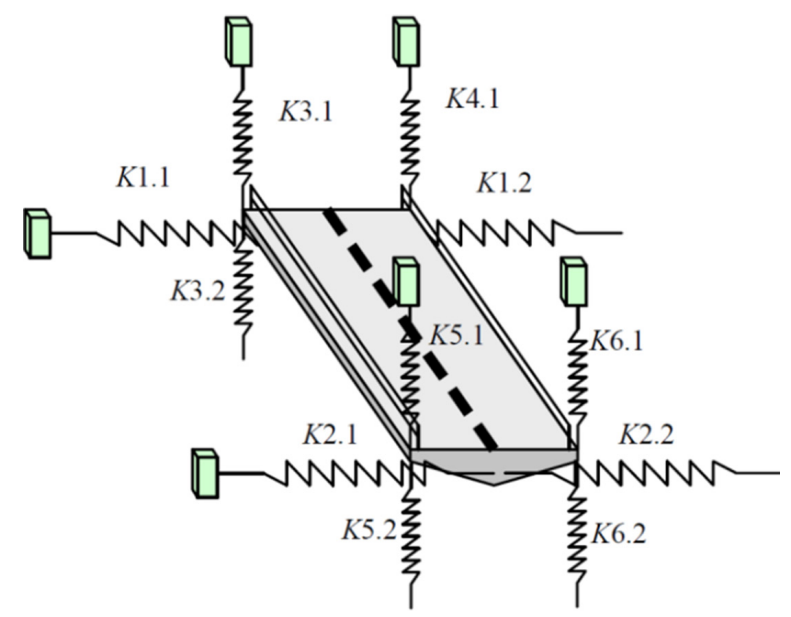

Figure 10: Setup of the aeroelastic tests.

allow the movement required for the space needed, and the stiffness has to be adequate to obtain the correct natural frequencies. These frequencies along with the wind speed $U$ and the sectional model width $B$ determine the rank of reduced velocities $U^{*}$ defined in eqn (2), where it is possible to obtain the flutter derivatives.

$$
U^{*}=\frac{2 \pi U}{\omega B} .
$$

Flutter derivatives were obtained for two different setups. Firstly, to acquire the flutter derivatives for the windward position (single), only one row of panels was tested. Secondly, two sectional models were situated in the test chamber; the windward model was fixed without displacements, while the leeward model was supported by springs and tested to obtain the flutter derivatives for the leeward position (wake). Figure 11 shows this last case.
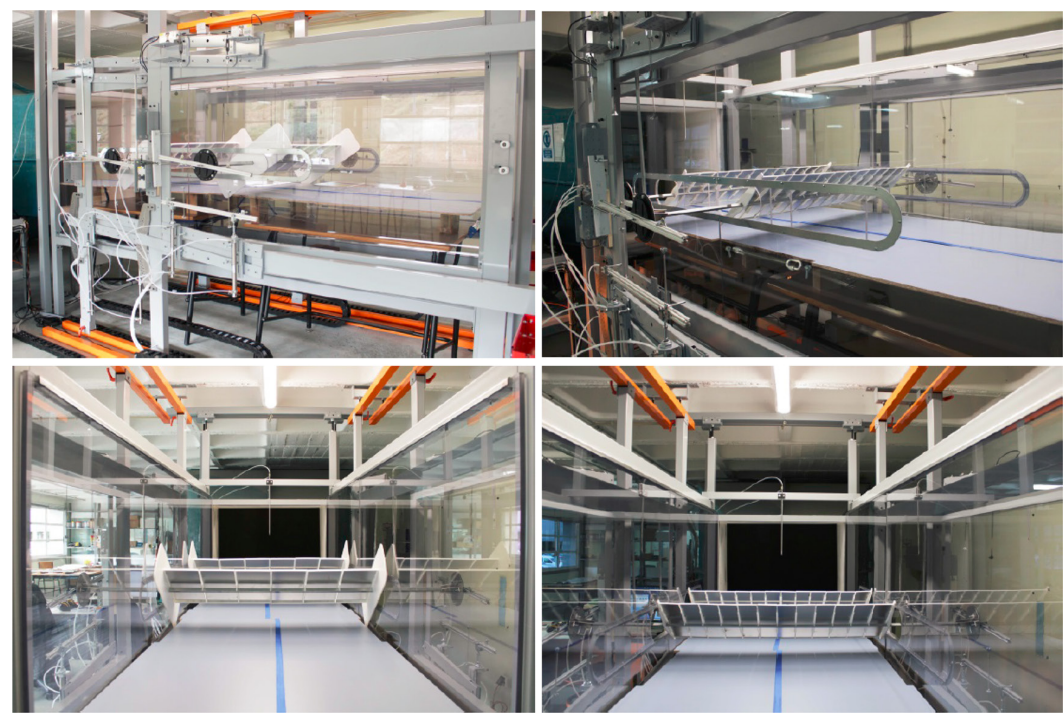

Figure 11: Wake setup of the solar tracker for configuration 1 (left) and configuration 2 (right). 
$A_{i} *$
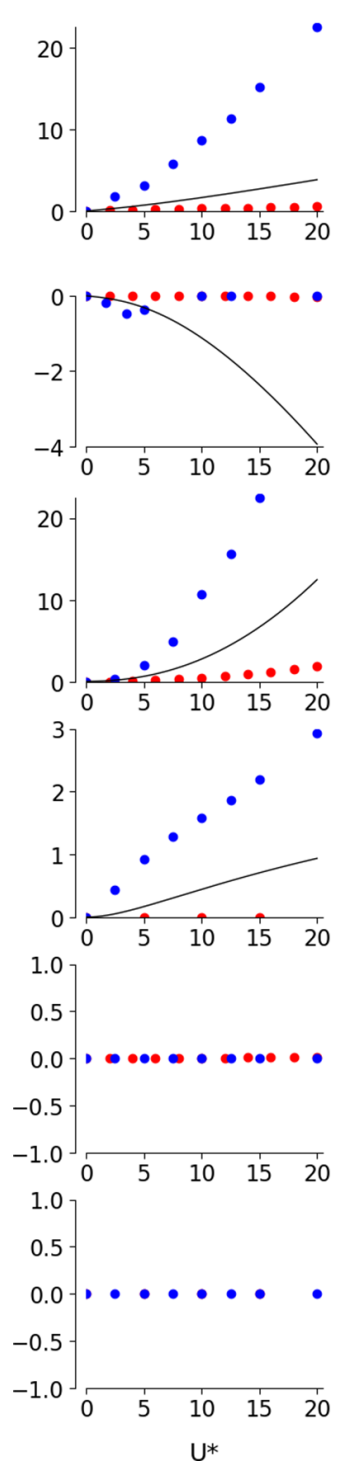

$H_{i}{ }^{*}$
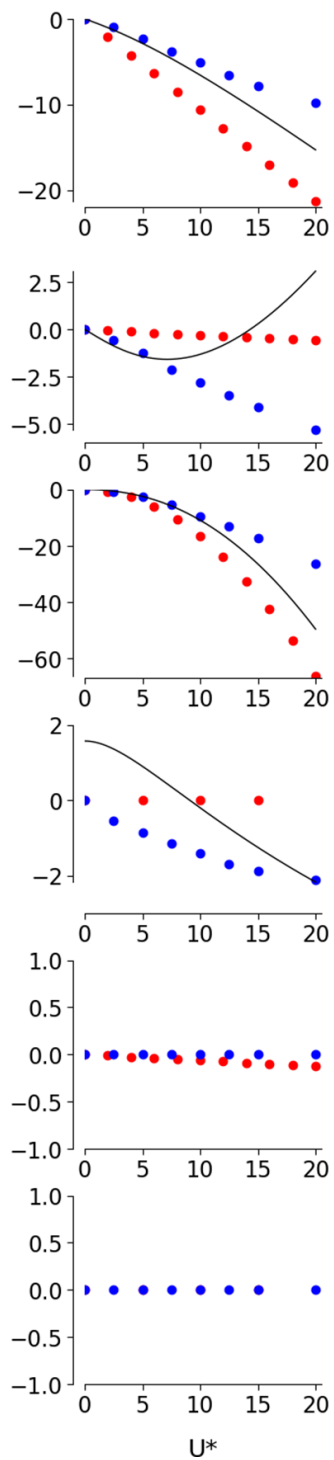

$P_{i} *$
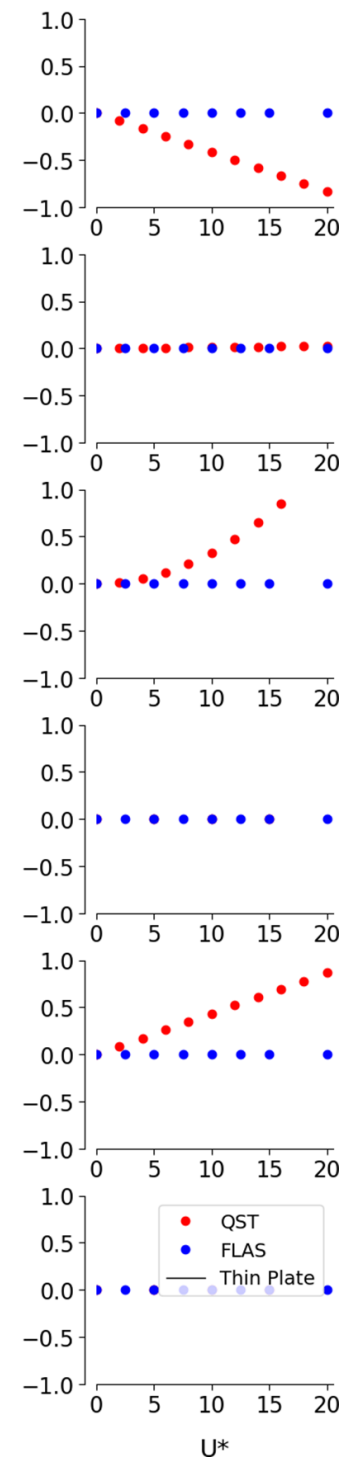

Figure 12: Comparison of the values of the flutter derivatives used in code FLAS (blue), the values obtained in quasi-steady theory (red), and the thin plate (black).

A post-processing of the values obtained in the wind tunnel was carried out to introduce the values of the flutter derivatives in code FLAS [34], reducing the number of points used to define the tendency of the curve. In addition, the flutter derivatives were calculated from the aerodynamic coefficients using quasi-steady theory [35]. Figure 12 shows a comparison between the values of the flutter derivatives used in FLAS, the values obtained by quasi-steady theory, and the theoretical values of a thin plate. Flutter derivatives $A_{i}^{*}, H_{i}^{*}, P_{i}^{*}$ $(i=1, \ldots, 6)$ are presented in the columns. Thus, each row contains the values of one $A_{i}{ }^{*}, H_{i}{ }^{*}$, $P_{i}{ }^{*}$ flutter derivative with $A_{1}{ }^{*}, H_{1}{ }^{*}, P_{1}{ }^{*}$ at the top and $A_{6}{ }^{*}, H_{6}{ }^{*}, P_{6}{ }^{*}$ at the bottom. 


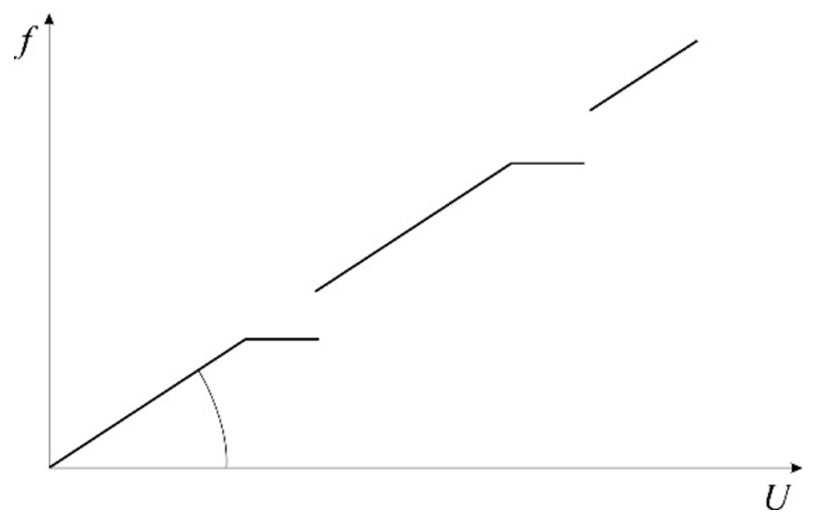

Figure 13: Relationship between the vortex frequency and the wind speed and lock-in phenomenon.

\subsection{Vortex shedding test}

A bluff body immersed in a fluid alters the flow and generates a sequence of vortices that shed alternatively downwind the body; the frequency of these vortices is related to the wind speed $U$ by eqn (3), where $S_{t}$ is the Strouhal number and $D$ is a characteristic dimension of the body. These vortices produce across wind forces, and consequently, body vibrations and wind displacement of a small value, but if the frequency of the vortices approaches a natural frequency of the body, the frequency of the vortex does not change even with increments in the wind speed. This phenomenon is known as lock-in.

Figure 13 shows the behavior of the bluff body; the inclined line with slope $S_{t} / D$ represents the situations when the vortex frequency varies linearly with the wind speed and the horizontal lines represent the lock-in events, the intervals of wind speed in which the vortex frequency does not change and maintains the value of a natural frequency y of the bluff body:

$$
S_{t}=\frac{f D}{U}
$$

The coupling of vortex frequency to the natural frequency of the body remains during a range of wind flow different in each body, and during this interval, the displacements are very relevant and may produce fatigue-related problems in the body. Therefore, evaluation of the Strouhal number and characterization of the wind speed $U$ that can produce the lock-in phenomenon are very important. In this research, the vortex shedding study was carried out on the solar tracker of the configuration 1 . The reduced model of a segment of the solar tracker tested in TUVA had a geometric scale of $1 / 6.25$, so it had the dimensions presented in Table 4 and was installed rigidly fixed to both sides of the test chamber.

It is well known that the Strouhal number depends on the value of the Reynolds $R_{e}$ number; so, the test needed to be carried out for a number of speeds of wind flow until the value of the

Table 4: Dimensions of the reduced model.

\begin{tabular}{llll}
\hline$B(\mathbf{m})$ & $D(\mathbf{m})$ & $W(\mathbf{m})$ & $S\left(\mathbf{m}^{2}\right)$ \\
\hline 0.32 & 0.030 & 1.00 & 0.32 \\
\hline
\end{tabular}



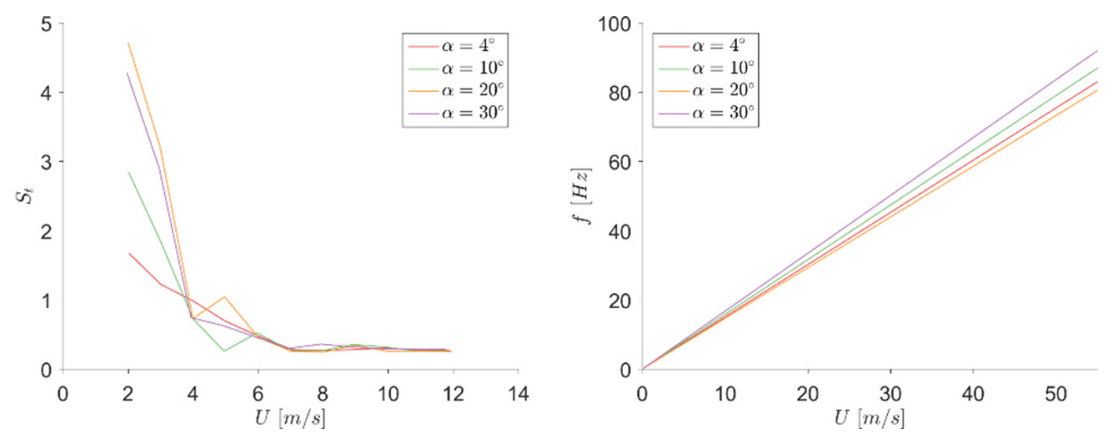

Figure 14: Evolution of the Strouhal number for $\alpha=4^{\circ}, 10^{\circ}, 20^{\circ}, 30^{\circ}$ (left) and relationship between vortex shedding frequency and wind flow speed (right).

Table 5: Values of $S_{t}$.

\begin{tabular}{lllll}
\hline$\alpha$ & $4^{\circ}$ & $10^{\circ}$ & $20^{\circ}$ & $30^{\circ}$ \\
$S_{t}$ & 0.269 & 0.270 & 0.256 & 0.283 \\
\hline
\end{tabular}

$S_{t}$ converged. Thus, a range of wind speeds $U$ (between 1 and $12 \mathrm{~m} / \mathrm{s}$ ) was applied at intervals of $1 \mathrm{~m} / \mathrm{s}$. The test was carried out for four inclinations of the reduced models, namely $\alpha=4^{\circ}$, $10^{\circ}, 20^{\circ}, 30^{\circ}$. The TUVA tunnel has cell loads that allow one to obtain the vertical force $L$. The experiment started with the identification of the vertical force (lift) that is related to the aerodynamic coefficient $C_{L}$ as explained in eqn (1). The test consisted of the following steps for each value of wind speed:

1. recording of time history of the lift $L$ and thus $C_{L}$ using eqn (1);

2. calculation of the power spectral density (PSD) of $C_{L}$;

3. filtering of the PSD to eliminate the natural frequencies of the model; and

4. identification of the frequency that corresponds to the peak frequency of the PSD.

Figure 14 shows the evolution in the range of wind speeds studied, and it can be observed that convergence appears for values higher than $U=8 \mathrm{~m} / \mathrm{s}$. The Strouhal number of the panels for each inclination appears in Table 5. Once the Strouhal number $S_{t}$ is obtained, it is possible to represent the relationship between the frequency of the vortex in the real panel and wind speed using eqn (3) because $S_{t}$ has the same value in the model used in the test and in the real structure and it is presented in Fig. 14. The lock-in phenomenon can appear when the vortex shedding corresponds to one of the natural frequencies presented in Table 2.

\section{STUDY OF TORSIONAL DIVERGENCE}

\subsection{Description of the torsional divergence phenomenon}

Torsional divergence, sometimes entitled aerostatic stability, is a well-known aerodynamic effect produced by wind-induced loads that increase monotonically the torsional gyration and produce collapse of the structure. The phenomenon was studied for the first time in aircraft wings, and only the twisting moment generated by wind flow was included in the analysis. 

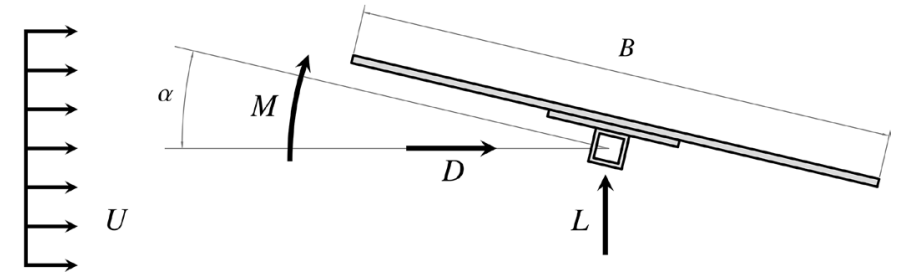

Figure 15: Sign convention of wind forces.

More recently, this phenomenon was of interest in other slender structures such as long span bridges, and in this case, the three forces ( $L$, lift, $D$, drag, and $M$, moment) created by wind were included in the analysis and are expressed by eqn (1). Sign convention appears in Fig. 15.

\subsection{Torsional divergence study of the solar tracker}

The solar tracker of the configuration 1 fabricated by STI Norland was used in this study. The structural model appears in Fig. 16; it was created with bar elements and the longitudinal axis has 243 nodes. The wind forces used the values of the aerodynamic coefficients obtained in the wind tunnel test described previously. The aerodynamic forces acting in the nodes of the longitudinal axis compose the forces vector $\mathbf{p}$. Recalling nodal decomposition methods of dynamic analysis, it is well known that the displacement vector $\mathbf{u}$ of a structure can be given as:

$$
\mathbf{u}=\sum_{n=1}^{n} \mathbf{u}_{n} .
$$

where $\mathbf{u}$ is the total displacement and $\mathbf{u}_{n}$ is the component of the $n$-esime node that can be obtained [36] as:

$$
\mathbf{u}_{n}=\frac{\Gamma_{n}}{\omega_{n}^{2}} \phi_{n} .
$$

where $f_{n}$ is the $n$-th eigenvector, $w_{n}$ is the $n$-th natural frequency, and $\mathbf{G}_{n}$ is as presented in eqn (6) when the eigenvectors are mass normalized:

$$
\Gamma_{n}=\phi_{n}^{T} \mathbf{p}
$$

Carrying out the proper algebraic substitutions, it turns out that:

$$
\mathbf{u}=\sum_{n=1}^{n} \frac{\phi_{n}^{T} \mathbf{p} \phi_{n}}{\omega_{n}^{2}}
$$

The procedure to observe the behavior of the solar tracker is an iterative one that was carried out for four inclinations of the panel, namely, $4^{\circ}, 10^{\circ}, 20^{\circ}, 30^{\circ}$. Wind speeds considered in the analysis were $15,30,45$, and $55 \mathrm{~m} / \mathrm{s}$. The steps of the procedure are as follows:

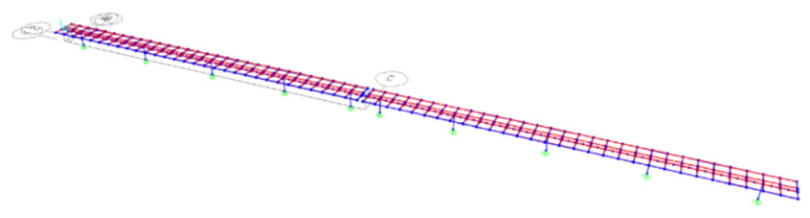

Figure 16: Structural model of the solar tracker. 


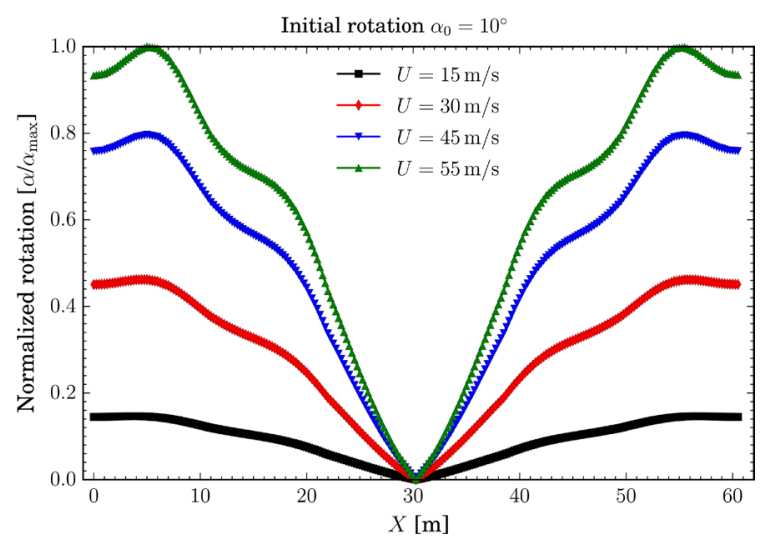

Figure 17: Values of torsional rotation along the panel for $\alpha=10^{\circ}$.

1. The forces corresponding to the initial inclination $a_{0}$ are applied on each node.

2. A static structural analysis is carried out and the vector of displacement gives the angle $a_{i}$ at each node, that is, $a_{i}=a_{0}+a_{1 \mathrm{i}}(i=1, \ldots, N)$, with $a_{1 \mathrm{i}}$ being the rotation produced by the forces at the $i$-th node.

3. The new loads at each node corresponding to the inclination $a_{i}$ are calculated and applied to the structural model. The outcome are the angles $a_{2 \mathrm{i}}$ and $a_{i}=a_{0}+a_{2 \mathrm{i}}(i=1, \ldots, N)$.

4. Steps 2 and 3 are carried out several times until obtaining the convergence of values $a_{i}$ $(i=1, \ldots, N)$ in two consecutive iterations.

If convergence does not occur, it means the wind speed $U$ will produce torsional divergence in this panel. The panel used in the study behaved very properly for that range of speeds and no instability appeared. Figure 17 shows some information on rotations for an initial inclination of $10^{\circ}$.

\section{COMPUTATIONAL ANALYSIS OF FLUTTER}

Flutter phenomena are analyzed considering the dynamic equilibrium equation considering self-excited forces that can be written as:

$$
\mathbf{M u}+\mathbf{C} \dot{\mathbf{u}}+\mathbf{K u}=\mathbf{f}_{a} .
$$

where $\mathbf{M}, \mathbf{C}$, and $\mathbf{K}$ are the mass, damping, and stiffness matrix, respectively, and $\mathbf{u}, \dot{\mathbf{u}}$ and $\ddot{\mathbf{u}}$ are the displacement, velocity, and acceleration vectors of the structure, respectively. In order to include the effect of mechanical dampers, a new mechanical damping matrix $\mathbf{C}_{m}$ is defined.

$$
\mathbf{f}_{m, i}=\left(\begin{array}{l}
F_{x} \\
F_{y} \\
F_{z} \\
M_{x} \\
M_{y} \\
M_{z}
\end{array}\right)_{i}=\left(\begin{array}{cccccc}
c_{u} & 0 & 0 & 0 & 0 & 0 \\
0 & c_{v} & 0 & 0 & 0 & 0 \\
0 & 0 & c_{w} & 0 & 0 & 0 \\
0 & 0 & 0 & c_{\varphi x} & 0 & 0 \\
0 & 0 & 0 & 0 & c_{\varphi y} & 0 \\
0 & 0 & 0 & 0 & 0 & c_{\varphi z}
\end{array}\right)\left(\begin{array}{l}
\dot{u} \\
\dot{v} \\
\dot{w} \\
\dot{\varphi}_{x} \\
\dot{\varphi}_{y} \\
\dot{\varphi}_{z}
\end{array}\right)=\mathbf{C}_{m} \dot{\mathbf{u}}_{i} .
$$

where $c_{\varphi}$, the damper constant, is the relationship between the torsional moment and the angular velocity. Figure 18 shows a scheme of the damper. 


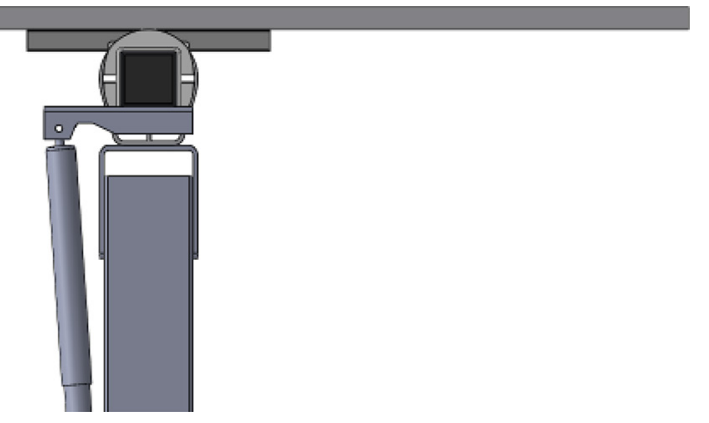

Figure 18: Damper scheme.

Knowing the damping constant and the distance $a$ from the damper to the axis of the solar tracker, the value of $c_{\varphi}$ can be obtained from the nominal value of the damper $c_{w}$ as:

$$
M_{x}=F a=c_{\varphi} \dot{\varphi}_{x}=c_{\varphi} \frac{\dot{w}}{a} \Rightarrow F=\frac{c_{\varphi}}{a^{2}} \dot{w}=c_{w} \dot{w} \Rightarrow c_{\varphi}=c_{w} a^{2}
$$

Introducing the new matrix $\mathbf{C}_{m}$ in eqn (8), the system of equations governing the dynamic behaviour while withstanding forces of aeroelastic origin is obtained:

$$
\mathbf{M u ̈}+\left(\mathbf{C}+\mathbf{C}_{\mathbf{m}}-\mathbf{C}_{\mathbf{a}}\right) \dot{\mathbf{u}}+\left(\mathbf{K}-\mathbf{K}_{\mathbf{a}}\right) \mathbf{u}=\mathbf{0},
$$

where matrix $\mathbf{C}$ contains the classical structural damping ratio, matrix $\mathbf{C}_{m}$ links the forces that the mechanical dampers apply with the velocities of the nodes where the forces act, and matrix $\mathbf{C}_{a}$ represents the aeroelastic damping. Equation (11) can be solved as an eigenvalue problem. The solution is $(i=1, \ldots, 2 m)$, where $\alpha$ is related to structural damping and $\beta$ is the damping frequency. An iterative procedure is necessary to obtain the eigenvalues, and the critical flutter speed occurs when an eigenvalue with a real part null is found.

Several analyses were conducted with different combinations of natural modes. The analyses were performed with a damping of $2 \%$ for the lateral modes $\left(\xi_{v}\right), 3 \%$ for the vertical modes $\left(\xi_{w}\right)$, and $10 \%$ for the torsional modes $\left(\xi_{\phi}\right)$. These values of damping were verified with a testing campaign for such purpose over a real-scale tracker prototype. Both configurations were analyzed for all the angles of attack considered and for the cases of activated and non-activated mechanical dampers. The damping constant of the mechanical dampers considered was $40 \mathrm{kN} \mathrm{s} / \mathrm{m}$. Results of the critical flutter speed obtained with software FLAS in all the analyses normalized to the critical flutter speed for $0^{\circ}$ are shown in Table 6 .

In addition, the critical flutter speed of the configuration 1 was calculated using a different software called Dinasolar developed by STI Norland. Table 7 shows a comparison between the values obtained with both software programs normalized to the critical speed for $0^{\circ}$.

\section{CONCLUSIONS}

This paper contains a comprehensive study, both experimental and computational, of two configurations of solar trackers composed of rows of 30 panels, which was aimed to verify their structural and dynamic behavior under a number of wind-induced phenomena, namely, vortex shedding, torsional divergence, and flutter. The experimental studies have been carried out using reduced models of the panels in a wind tunnel. 
Table 6: Critical flutter speed normalized (m/s).

\begin{tabular}{llllll}
\hline & & & Configuration 1 & \multicolumn{1}{c}{ Configuration 2 } \\
\hline$\xi_{\phi}(\%)$ & & 10 & 10 & 10 & 10 \\
$\xi_{w}(\%)$ & & 3 & 3 & 3 & 3 \\
$\xi_{v}(\%)$ & 2 & 2 & 2 & 2 \\
$c_{\text {dampers }}(\mathrm{kN} \mathrm{s} / \mathrm{m})$ & & 0 & 40 & 0 & 40 \\
& $0^{\circ}$ & 1.0 & 1.0 & 1.0 & 1.0 \\
& $10^{\circ}$ & 1.1 & 1.1 & 1.2 & 1.0 \\
& $20^{\circ}$ & 10.7 & 14.6 & 7.5 & 8.1 \\
& $35^{\circ}$ & 11.6 & 13.9 & 12.4 & 12.5 \\
Angle of attack & $60^{\circ}$ & 14.8 & 25.9 & 16.0 & 19.3 \\
& $-10^{\circ}$ & 1.1 & 1.0 & 1.1 & 1.1 \\
& $-20^{\circ}$ & 1.2 & 1.1 & 1.2 & 1.2 \\
& $-35^{\circ}$ & 1.0 & 1.1 & 2.1 & 2.2 \\
& $-60^{\circ}$ & 9.9 & 9.1 & 6.2 & 6.3 \\
\hline
\end{tabular}

Table 7: Comparison of the critical flutter speed between software programs.

\begin{tabular}{llll}
\hline Angle of attack & FLAS & Dinasolar & Variation $(\%)$ \\
\hline $0^{\circ}$ & 1.00 & 1.06 & 6.08 \\
$10^{\circ}$ & 1.11 & 1.09 & -1.22 \\
$20^{\circ}$ & 10.70 & 10.00 & -6.68 \\
$35^{\circ}$ & 11.58 & 11.89 & 2.68 \\
$60^{\circ}$ & 14.76 & 16.00 & 8.42 \\
$-10^{\circ}$ & 1.05 & 1.02 & 3.21 \\
$-20^{\circ}$ & 1.19 & 1.23 & 3.41 \\
$-35^{\circ}$ & 0.99 & 1.08 & 9.59 \\
$-60^{\circ}$ & 9.95 & 10.14 & 8.42 \\
\hline
\end{tabular}

The tests with a reduced model rigidly fixed to the walls of the test chamber have allowed one to identify the Strouhal number, $S_{t}$, and its dependence on the Reynolds number, $R_{e}$. The numerical results in the test with increasing values of $R_{e}$ have shown a monotonic variation that is a symptom of absence of lock-in phenomenon for the range of wind speeds considered. Thus, it can be concluded that no incidence due to vortex-induced vibrations will appear in the solar trackers for the range of angle orientations and wind speeds considered in the study.

The torsional divergence study started by identifying the aerodynamic coefficients and the forces generated by wind and continued by carrying out structural analysis using a finite element model of the full solar tracker subject to these loads. A wide range of angles of attack of the flow and values of wind speed were taken into account, and the results showed a 
complete stability of the design up to very high values of wind speed $(55 \mathrm{~m} / \mathrm{s})$. Therefore, the solar trackers are not subjected to torsional divergence instability.

The flutter study initiated with a complex experimental setup that allowed one to allocate simultaneously two reduced models of the panels in the test chamber in order to identify the complete set of the 18 flutter derivatives of the upwind and downwind models. After that, a computational study was carried out using a finite element model subjected to the aeroelastic forces that are functions of the flutter derivatives. Again, several values of the damping generated by the damper device located in the longitudinal axis of the solar tracker and a vast interval of the angle of attack of the wind flow were considered. The resultant nonlinear eigenvalue problem was solved using the code FLAS that provided the value of the flutter speed and the dependence of the inclination of the solar tracker for each case studied. A comparison of these results with those generated with software Dinasolar developed by STI Norland, showing differences less that $10 \%$, is also presented.

\section{REFERENCES}

[1] Simiu, E. \& Scanlan, R.H., Wind effects on structures. Fundamentals and Applications to Design. Wiley \& Sons: New York, Chichester, Brisbane, Toronto and Singapore, 1996.

[2] Dyrbye, C. \& Hansen, S.O., Wind loads on structures. Fundamentals and Applications to Design. Wiley \& Sons: Chichester, New York, Brisbane, Toronto and Singapore, 1997.

[3] Bibin, C., Selvaraj, M.J. \& Sanju, S., Flutter analysing over an aircraft wing during cruise speed. Procedia Engineering, 38, pp. 1950-1961, 2012. https://doi.org/10.1016/ j.proeng.2012.06.238

[4] Shubov, M.A., Flutter phenomenon in aeroelasticity and its mathematical analysis. Journal of Aerospace Engineering, 19(1), pp. 1-12, 2006. https://doi.org/10.1061/ (asce)0893-1321(2006)19:1(1)

[5] Yoon, N.K., Chung, C.H., Na, Y.-H. \& Shin, S.J., Control reversal and torsional divergence analysis for a high-aspect-ratio wing. Journal of Mechanical Science and Technology, 26(12), pp. 3921-3931, 2012. https://doi.org/10.1007/s12206-012-0889-2

[6] Bruce Ralphin Rose, J. \& Jinu, G.R. Influence of aeroelastic control reversal problem in the airplane lateral stability modes. Proceedings of the Institution of Mechanical Engineers, Part G: Journal of Aerospace Engineering, 229(3), pp. 517-533, 2015. https:// doi.org/10.1177/0954410014537241

[7] Mondal, T., Das, M.K. \& Guha, A. Periodic vortex shedding phenomenon for various separation distances between two plane turbulent parallel jets. International Journal of Heat and Mass Transfer, 99, pp. 576-588. 2016.

[8] Sikien, E.R., Abdullah, A., Zulkafli, M.F. \& Rahim, M.Z. The effects of vortex shedding on the aerodynamic performance of airfoils. ARPN Journal of Engineering and Applied Sciences, 13(24), pp. 9344-9351, 2018.

[9] Scott, R., In the Wake of Tacoma. Suspension Bridges and the Quest for Aerodynamic Stability. ASCE Press: USA, 2001.

[10] Scanlan, R.H., Studies of suspension bridge deck flutter instability. CASI/AIAA Subsonic Aero-and Hydro-Dynamics Meeting, p. 9, 1969.

[11] Larsen, A., Aerodynamic aspects of the final design of the $1624 \mathrm{~m}$ suspension bridge across the Great Belt. Journal of Wind Engineering and Industrial Aerodynamics, 48(2-3), pp. 261-285, 1993. 
[12] Jurado, J.A. \& Hernández, S., Sensitivity analysis of bridge flutter with respect to mechanical parameters of the deck. Structural and Multidisciplinary Optimization, 27(4), pp. 272-283, 2004. https://doi.org/10.1007/s00158-003-0374-8

[13] Larsen, A. \& Wall, A., Shaping of bridge box girders to avoid vortex shedding response. Journal of Wind Engineering and Industrial Aerodynamics, 104-106, pp. 159-165, 2012. https://doi.org/10.1016/j.jweia.2012.04.018

[14] Yuan, W., Laima, S., Chen, W., Li, H. \& Hu, H., Investigation on the vortex-and-wakeinduced vibration of a separated-box bridge girder. Journal of Fluids and Structures, 70, pp. 145-161, 2017.

[15] Laima, S., Li, H., Chen, W., \& Ou, J., Effects of attachments on aerodynamic characteristics and vortex-induced vibration of twin-box girder. Journal of Fluids and Structures, 77, pp. 115-133, 2018. https://doi.org/10.1016/j.jfluidstructs.2017.12.005

[16] Boonyapinyo, V., Lauhatanon, Y. \& Lukkunaprasit, P., Nonlinear aerostatic stability analysis of suspension bridge. Engineering Structures, 28, pp. 793-803, 2006. https:// doi.org/10.1016/j.engstruct.2005.10.008

[17] Cheng, J., Jiang, J., Xiao, R.C, \& Xiang, H.F., Nonlinear aerostatic stability analysis Jiang Yin suspension bridge. Engineering Structures, 24, pp. 773-781, 2002. https:// doi.org/10.1016/s0141-0296(02)00006-8

[18] Qian, C. \& Zhu, L. Study on the mechanism of aerostatic instability of long-span cablestayed bridges. In the 15th Int. Conf. on Wind Eng. Beijing, China, 1-6 September 2019.

[19] Jonsson, E., Riso, C., Lupp, C.A., Cesnik, C.E.S., Martins, J.R.R.A. \& Epureanu, B.I., Flutter and post-flutter constraints in aircraft design optimization. Progress in Aerospace Sciences, 109, Art. No. 100537, 2019.

[20] Dillinger, J., Abdalla, M., Klimmek, T. \& Gürdal, Z., Static Aeroelastic Stiffness Optimization and Investigation of Forward Swept Composite Wings. 10th World congress on structural and multidisciplinary optimization, Orlando, FL, 2013.

[21] Kusano, I., Baldomir, A., Jurado, J.A. \& Hernandez, S., Reliability based design optimization of long-span bridges considering flutter. J. Wind Eng. Ind. Aerod., 135, pp. 149-162, 2014. https://doi.org/10.1016/j.jweia.2014.10.006

[22] Cid Montoya, M., Hernandez, S., \& Nieto, F., Shape optimization of streamlined decks of cable-stayed bridges considering aeroelastic and structural constraints. J. Wind Eng. Ind. Aerod., 177, pp. 429-455, 2018. https://doi.org/10.1016/j.jweia.2017.12.018

[23] Cid Montoya, M., Hernandez, S., Nieto, F., Kareen, A. \& Jurado, J.A. Aerostructural shape and size optimization of a long span cable stayed bridge considering service loads, static wing loads, torsional divergence, flutter and buffeting constraints. In the 15th Int. Conf. on Wind Eng. Beijing, China, 2-6 September 2019.

[24] Jurado, J.A., Hernández, S., Nieto, F. \& Mosquera, A., Bridge Aeroelasticiy. Sensitivity Analysis and Optimal Design. WIT Press: Ashurst Lodge, Ashurst. Southampton, 2011.

[25] Rizk, J. \& Chaiko, Y., Solar Tracking System: More Efficient Use of Solar Panels. World Academy of Science, Engineering and Technology 41, 2008.

[26] Vieira, R.G., Guerra, F.K.O.M.V., Vale, M.R.B.G. \& Araújo, M.M., Comparative performance analysis between static solar panels and single-axis tracking system on a hot climate region near to the equator. Renewable and Sustainable Energy Reviews, 64, pp. 672-681, 2016. https://doi.org/10.1016/j.rser.2016.06.089

[27] Aziz, S. \& Hassan, S., On improving the efficiency of a solar panel tracking system. Procedia Manufacturing, 7, pp. 218-224, 2017. https://doi.org/10.1016/j.promfg. 2016.12.053 
[28] Racharla, S. \& Rajan, K., Solar tracking system-a review. International Journal of Sustainable Engineering, 10(2), pp. 72-81, 2017.

[29] Hernandez, S., Mendez, J., Nieto, F. \& Jurado, J.A., Aerodynamic analysis of a photovoltaic solar tracker. EACWE5. The Fifth European \& African Conference on Wind Engineering, Firenze, July 2009.

[30] Ibrahim, S.R., Fundamentals of Time Domain Modal Identification. In Silva, J.M.M., \& Maia N.M.M. eds., Modal Analysis and Testing. NATO Science Series (Series E: Applied Sciences), Vol. 363. Springer, Dordrecht, 1999. https://doi.org/10.1007/97894-011-4503-9_11

[31] Smith, W.R., Least squares time-domain method for simultaneous identification of vibration parameters from multiple free-response records. Proceedings of 22nd AIAA Structures, Structural Dynamics, and Materials Conference, Atlanta, Georgia, pp. 194 201, 1981.

[32] Chen, A., He, X. \& Xiang, H., Identification of 18 flutter derivatives of bridge decks. Journal of Wind Engineering and Industrial Aerodynamic, 90, pp. 2007-2022, 2002. https://doi.org/10.1016/s0167-6105(02)00317-3

[33] Diana, G., Resta, F., Zasso, A., Belloli, M. \& Rocchi, D., Forced motion and free motion aeroelastic tests on a new concept dynamometric section model of the Messina suspension bridge. Journal of Wind Engineering and Industrial Aerodynamic, 92(6), pp. 441-462, 2004. https://doi.org/10.1016/j.jweia.2004.01.005

[34] Jurado, J.Á., Kusano, I., Hernández, S. \& Nieto, F., Improvement of multimodal flutter analysis code, FLAS. EACWE. Proc. European and African Congress in Wind Engineering Cambridge, UK, 7-11 July, 2013.

[35] Øiseth, O., Rönnquist, A. \& Sigbjörnsson, R., Simplified prediction of wind-induced response and stability limit of slender long-span suspension bridges, based on modified quasi-steady theory: A case study. Journal of Wind Engineering and Industrial Aerodynamics, 98(12), pp. 730-741, 2010. https://doi.org/10.1016/j.jweia.2010.06.009

[36] Chopra, A.K., Dynamic of structures. Theory and Applications to Earthquake Engineering, Prentice Hall: New Jersey, 1995. 